\title{
MONITORING OF THE EURASIAN NATURAL MINERAL WATERS OF CHLORIDE-HYDROCARBONATE TYPE
}

\author{
E.N. Kornilova, N.I.Kornilov*, O.N Vasiltseva. \\ Stavropol State Agrarian University, Stavropol, Russia \\ Email: nkornilov@26.ru, phone:+7-9034168841,fax:+7-8652263449
}

\begin{abstract}
Mineral waters of Russia, the CIS countries (Ukraine, Belarus, Moldova, Armenia, Azerbaijan, Georgia and Uzbekistan) and EU (Germany, France, Italy, Spain and some East European countries) carried to ChlorideHydrocarbonate type have been investigated in this work. The analytical dependences connecting, entered by authors, characteristic parameters of structure of waters $(\bar{Э}, B, \Phi, R)$ with independent variables of ionic structure and concentration are resulted.
\end{abstract}

Keywords: mineral waters, mathematical model, monitoring.

\section{Introduction}

Natural mineral waters concern to the major natural resources widely applied in medicine, food and a number of other branches of manufacture. The expansion of geography of developing deposits, extractions and pouring of mineral waters has led to the necessity of developing the system of monitoring and estimated parameters of quality of various waters on their origin and properties.

In this case the issue is on the development of mathematical model of water-salt systems of mineral waters on the basis of which it is possible to spend identification of regional mineral waters of various genesis and structure on their conformity to widely known (standard) mineral waters. Standard waters are presented to Russia in the state standard of GOST 13273-88 " Mineral, drinking, medical waters and medical table."

Now there are different ways of classification of mineral waters on a chemical compound. However the general model of classification of the mineral waters allowing quantitative estimation of conformity of regional mineral waters to reference waters of certain types, groups and classes has not been developed until now.

The purpose of this work consisted in the establishment of the quantitative dependences connecting certain estimated parameters with the mineralization and ionic structure of natural mineral waters of Chloride-Hydrocarbonate type. Mineral waters of Russia, the CIS countries and EU which are closest to the mineral waters included in State standard - GOST 13273-88 in terms of their ionic structure have been investigated in this work.

\section{Methodical part}

As the estimated parameters applied for the identification of waters of various origin and structure we had been introduced the representations about characteristic parameters of structure of waters and their interrelations with a chemical compound and the general mineralization of mineral waters.

As the initial parameters accepted for the mathematical description of structure of water-salt systems of mineral waters, following values have been entered:

1. $\bar{M}$ - The general mineralization of water, $\mathrm{g} / \mathrm{dm}^{3}$.

Value $\bar{M}$ is function of two independent variables $C_{N}$ and $X_{C{ }^{\prime}} / X_{H_{N O}^{\prime}}$, where $C_{N}$-equivalent (normal) concentration, eqv/dm ${ }^{3}$ and $X_{\mathrm{Cl}^{\prime}} / \mathrm{X}_{\mathrm{HNO}_{3}^{\prime}}$
water.

2. $\bar{\ni}$ - Gram-equivalent of salt system of mineral water, g/eqv, counted from a known parity in chemistry:

$$
\bar{M}=\bar{\Im} \cdot C_{N},
$$

The value $\bar{\Im}$ is accepted by us as the first characteristic parameter defining the type of mineral water and allowing to have investigated mineral waters of various ionic structures in the certain sequence.

The value $\bar{\jmath}$ can be calculated on the parity:

$$
\bar{\Im}=a\left(X_{C i} / X_{\mathrm{HNO}_{3}^{\prime}}\right)^{b},
$$

where $a$ and $b$ - are the constants corresponding to mineral waters of one type.

The estimation of an accessory of investigated water to the certain group of standard waters was performed by means of the function B, entered by us and connected with value $\bar{\Im}$ by the parity: 


$$
B=\frac{\bar{\ni}^{2}}{2}, \mathrm{~g}^{2} / \mathrm{eqv}^{2}
$$

Using the representations of quantum mechanics it is possible to assume, that the square of a characteristic parameter $\bar{\ni}$ of a solution is a measure of probability of conformity of an investigated solution to the some, are mainly determined (standard), to a solution.

In this case the square of a characteristic parameter $\bar{\ni}$ can be presented in the form of the second characteristic parameter which we have marked by symbol $B$.

In general the dependence of group value $B$ on the structure of solutions of the mineral waters forming the separate group of waters can be represented in the form of the parity:

$$
B=\frac{a^{2}}{2} \cdot\left(X_{C i} / X_{\mathrm{HNO}_{3}^{\prime}}\right)^{2 b}
$$

The analytical dependences presented by the equation (4) for two bordering groups on structure of waters allow solving a problem about the establishment of border of water division into different groups. Solving of this task is reduced to finding the value $X_{\mathrm{Cl}^{\prime}} / X_{\mathrm{HNO}_{3}^{\prime}}$ by joint solving of the system of two equations of the function $B$ on the parity:

$$
X_{\mathrm{Cl}^{\prime}} / X_{\mathrm{HNO}_{3}^{\prime}}=10^{\frac{\lg a^{\prime}-\lg a^{\prime \prime}}{b^{\prime \prime}-b^{\prime}}},
$$

where indexes ' and " - correspond to vicinal groups of mineral waters.

In order to estimate the belonging of separately taken mineral water to a certain classe of waters which are a part of group of standard waters, we have presented the third characteristic parameter $\Phi$ which represents the first derivative function $\mathrm{B}$ on argument ${ }^{\mathrm{Cl}^{\prime}} / \mathrm{X}_{\mathrm{HNO}_{3}^{\prime}}$.

Value $\Phi$ can be calculated from the parity:

$$
\Phi=y^{\prime}[B]=A\left(X_{C l} / X_{\mathrm{HNO}_{3}^{\prime}}\right)^{D},
$$

where $A$ and $D$ - the constants accordingly equal: $a^{2} \cdot b$ и $2 b-1$. The values A and D characterize the mineral waters with the close values of the value $X_{\mathrm{Ci}^{\prime}} / \mathrm{X}_{\mathrm{HNO}_{3}^{\prime}}$, forming a
separate class of certain group of mineral waters.

A logarithmic conversion of the equation (6) allows to receive the equation of linear regression and to continue processing the results by methods of mathematical statistics. In this work, the base computer program «Statistic-6» was applied.

The fourth characteristic parameter of mineral waters structure estimates the water-salt system in the form of function $R$ of two independent variables $C_{N}$ and $X_{C i} / X_{\mathrm{HNO}_{3}^{\prime}}$, which is the second derivative of function $\bar{M}$, described
by the equation (1).

The differentiation of function $\left[\bar{M}\left(C_{N}, \frac{X_{C l^{\prime}}}{X_{\mathrm{HCO}_{3}^{\prime}}}\right)\right]$, which is the complex function, has led to the parity:

$$
R=y^{\prime \prime}\left[\bar{M}_{i}\right]=[\bar{\ni}]^{\prime \prime}\left[C_{N}+\frac{2}{b-1}\left(\frac{X_{C l^{\prime}}}{X_{\mathrm{HCO}_{3}^{\prime}}}\right)\right] \text {, }
$$

where $[\bar{\ni}]^{\prime \prime}$ - the second derivative of value $\bar{\ni}$, corresponding expression

$$
[\bar{\ni}]^{\prime \prime}=a b(b-1)\left(\frac{X_{C l^{\prime}}}{X_{\mathrm{HNO}_{3}^{\prime}}}\right)^{(b-2)}
$$

Thus, the natural mineral waters corresponding to ascertained group of standard waters according to GOST 13273-88, should have strictly fixed quantitative characteristic parameters: $\bar{\ni}, B, \Phi$ and $R$, which will allow to estimate an accessory of investigated mineral water to the certain group ( $\bar{\ni}$ and $B$ ) and to a concrete class of waters of the given group on structure $(\Phi)$ and concentration $(R)[1]$. 


\section{Results and Discussion}

For natural Chloride-Hydrocarbonate waters included in XXII group of GOST 13273-88, we obtained the following dependences.

$$
\begin{aligned}
& \bar{\ni}=70,71\left({ }^{X_{\mathrm{Cl}}} / X_{\mathrm{HNO}_{3}^{\prime}}\right)^{-0,0762}, \\
& B=2498\left({ }^{X_{\mathrm{Cl}}} / X_{\mathrm{HNO}_{3}^{\prime}}\right)^{-0,1524}, \\
& \Phi=-381\left({ }^{X_{\mathrm{Cl}}^{\prime}} / X_{\mathrm{HNO}_{3}^{\prime}}\right)^{-1,1524}
\end{aligned}
$$

The analysis of the equations (9) - (11) has shown that mineral waters of the Chloride-Hydrocarbonate type, which belong according to GOST 13273-88 to XXII group, can be actually assigned to an independent group of waters characterized by group parameters $\bar{\ni}$ and $B$, according to equal values: 70,71 and $-0,0762$.

We established the borders of division of waters into groups between waters of Hydrocarbonate and ChlorideHydrocarbonate types, and also of Chloride-Hydrocarbonate and Chloride types on value $X_{\mathrm{Cl}^{\prime}} / X_{\mathrm{HNO}_{3}^{\prime}}$. Researches have shown, that the border of division in the first case is defined by the value $X_{\mathrm{Cl}^{\prime}} / X_{\mathrm{HNO}_{3}^{\prime}}$, equal to 0,218 and corresponds to the content of ions in a solution of 78,2\% of mg-eqv. In the second case these value are equal, accordingly: 3,624 and $21,6 \%$ of mg- eqv.

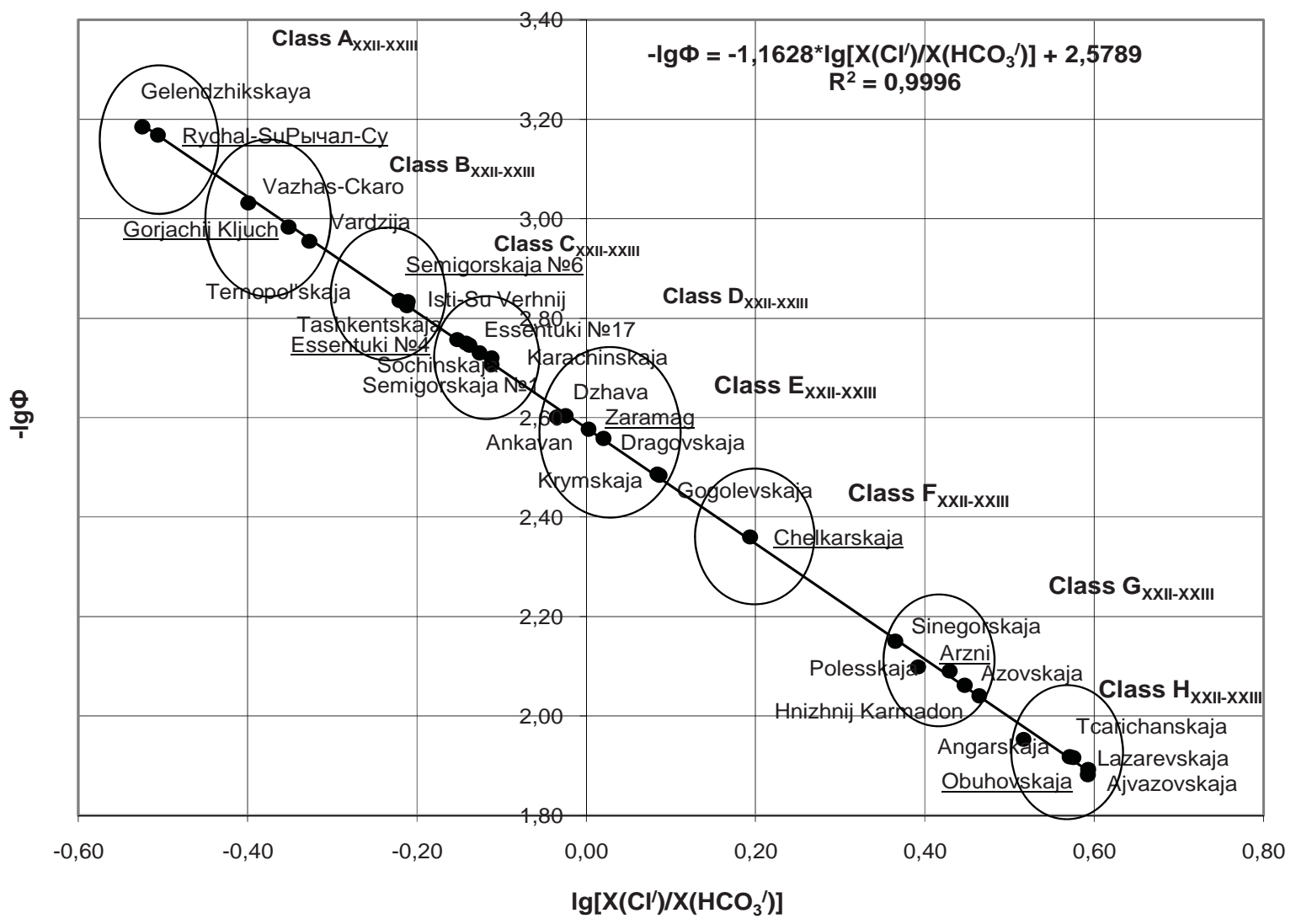

Figure 1. Graphic dependence of function “ $-\lg \Phi_{i} f\left[\lg \left(X_{\mathrm{Cl}^{i}} / \mathrm{X}_{\mathrm{HNO}_{3}^{\prime}}\right)_{i}\right]$ " ” for mineral waters XXII-XXIII of group according to the specified classification.

Figure 1 presents the graphic dependence of function " $-\lg \Phi_{i} f\left[\lg \left(\mathrm{X}_{\mathrm{Cl}} / \mathrm{X}_{\mathrm{HNO}_{3}^{\prime}}\right)_{i}\right]$ " " for standard waters of Chloride-Hydrocarbonate type, included in XXII- XXIII group (GOST 13273-88). 
Analytical expression of the equation of linear regress corresponds to expression

$$
\begin{aligned}
& -\lg \Phi_{i}( \pm 0,007)=-1,163( \pm 0,006) \lg \left(X_{\mathrm{Cl}^{\prime}} / X_{\mathrm{HNO}_{3}^{\prime}}\right)_{i}+2,579( \pm 0,002), \\
& R^{2}=0,9996
\end{aligned}
$$

From figure 1 follows, that separate representatives of mineral waters of Chloride-Hydrocarbonate type, assigned to XXII-XXIII groups, can be divided according to the size of the characteristic parameter $\Phi_{i}$ into half-groups (classes) in process of change of the value $\Phi_{i}$ :

1. The class $A$ - Rychal-Su (Russia), Gelendzhikskaja (Russia);

2. The class $B$ - Vazhas-Ckaro (Georgia), Gorjachij Kljuch (Russia), Vardzija (Georgia);

3. The class $C$ - Ternopol'skaja (Ukraine), Semigorskaja №6 (Russia), Isti-Su Verhnij (Azerbaijan),

4. The class $D$ - Tashkentskaja (Uzbekistan), Essentuki №4 and №17 (Russia), Karachinskaja (Russia), Semigorskaja №1 (Russia), Sochinskaja (Russia);

5. The class $E$ - Dzhava (Georgia), Ankavan (Armenia), Zaramag (Russia), Dragovskaja, Gogolevskaja and Krymskaja (Ukraine);

6. The class $F$ - Chelkarskaja (Kazakhstan);

7. The class $G$ - Polesskaja (Ukraine), Sinegorskaja, Azovskaja (Russia);

8. The class $H$ - Lazarevskaja, Obuhovskaja (Russia), Arzni (Armenia), Ajvazovskaja (Ukraine).

In figure 1, mineral waters inside a circle represent the separate subgroups (classes) generated on the basis of close values of value $X_{\mathrm{Cl}} / X_{\mathrm{HNO}_{3}^{\prime}}$.

Mineral waters are characterized within the limits of each class by the value of the characteristic parameter $R$, defining general mineralization (or concentration) of separately taken natural water.

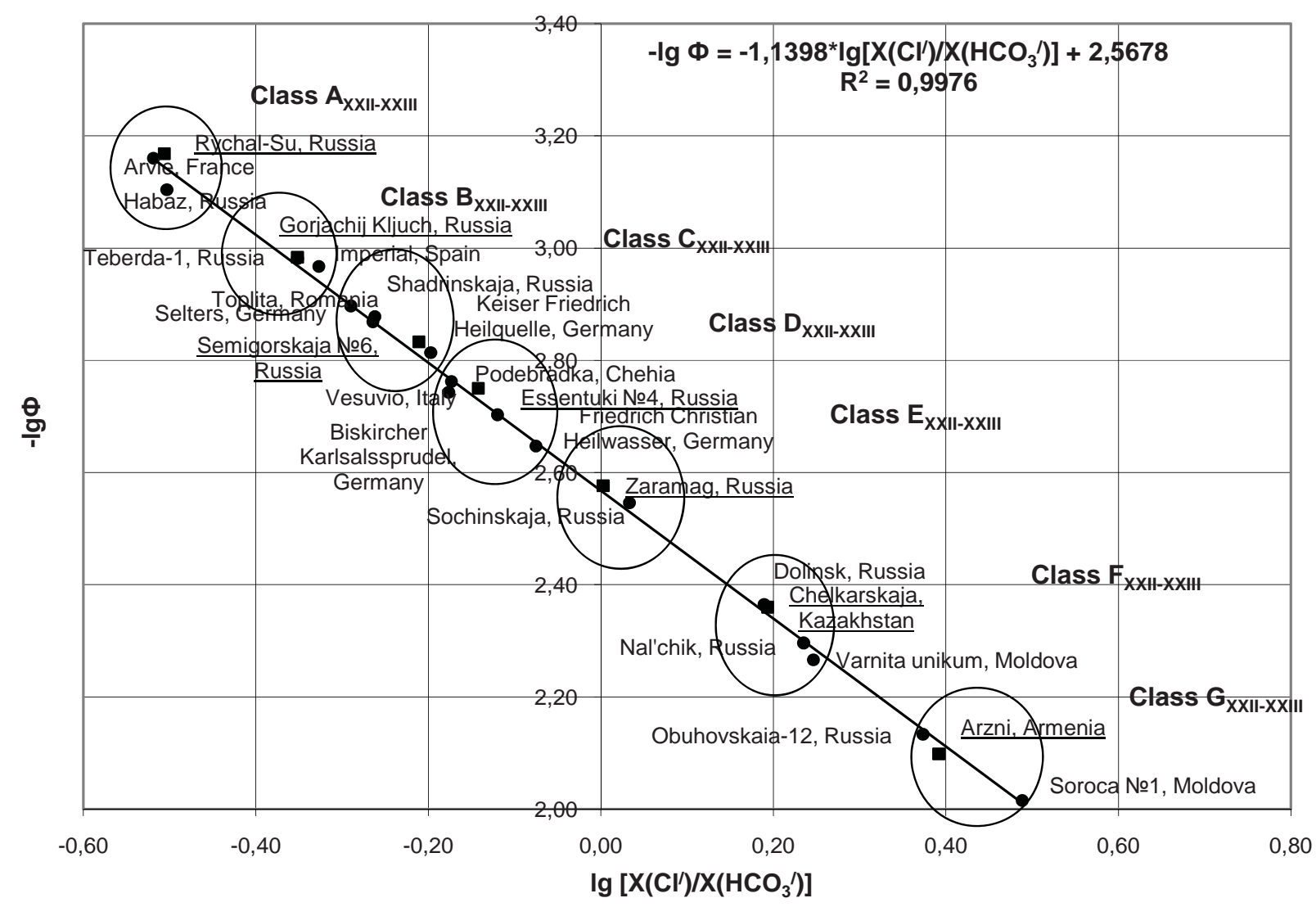

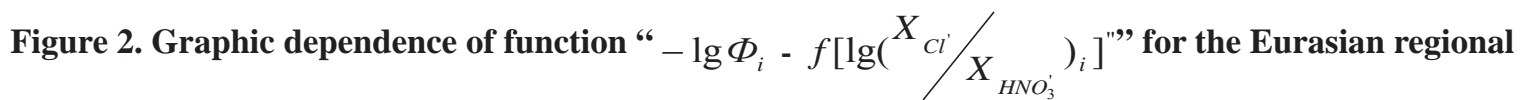
mineralized waters. 
In figures 2 and 3 are given the graphic dependences of logarithmic function " $-\lg \Phi_{i}-f\left[\lg \left(X_{\mathrm{Ci}} / \mathrm{X}_{\mathrm{HNO}_{3}^{\prime}}\right)_{i}\right]^{\prime \prime}{ }^{\prime}$ for regional Eurasian natural waters: mineral (fig.2) and waters with low mineralization with concentration less than $1 \mathrm{~g} / \mathrm{dm}^{3}$ (fig.3).

Analytical expressions of logarithmic functions for the specified waters correspond to the equations:

$$
\text { (2) }-\lg \Phi=-1,144 \lg \left(X_{\mathrm{Cl}^{\prime}} / \mathrm{X}_{\mathrm{HNO}_{3}^{\prime}}\right)+2,5685, R^{2}=0,9976
$$

and

$$
\text { (3) }-\lg \Phi=-1,1345 \lg \left(X_{C i} / X_{H_{N}^{\prime}}\right)+2,547, R^{2}=0,9962
$$

The comparison of values of factors $A$ and $D$ for the standard and Eurasian regional mineral waters given by the equations (12) - (14) has shown their full practical concurrence. The generalized statistical processing of the obtained data for the Eurasian and standard waters has shown, that the factor of correlation of the equations is in all cases within the limits of $r=0,995-0,999$ and the error of calculations did not exceed the standard deviations of $5 \%$-s' level of a confidential interval.

The analysis of settlement data of a characteristic parameter of structure $\Phi$ from analytical dependences (13) and (14) has shown, that natural mineral waters of different sources of the Eurasian continent, regardless of their geographical position, form families of waters with close values of ionic structure $X_{\mathrm{Cl}^{\prime}} / X_{\mathrm{HNO}_{3}^{\prime}}$, which testifies to unity of genesis of
water-containing layers and mineral waters.

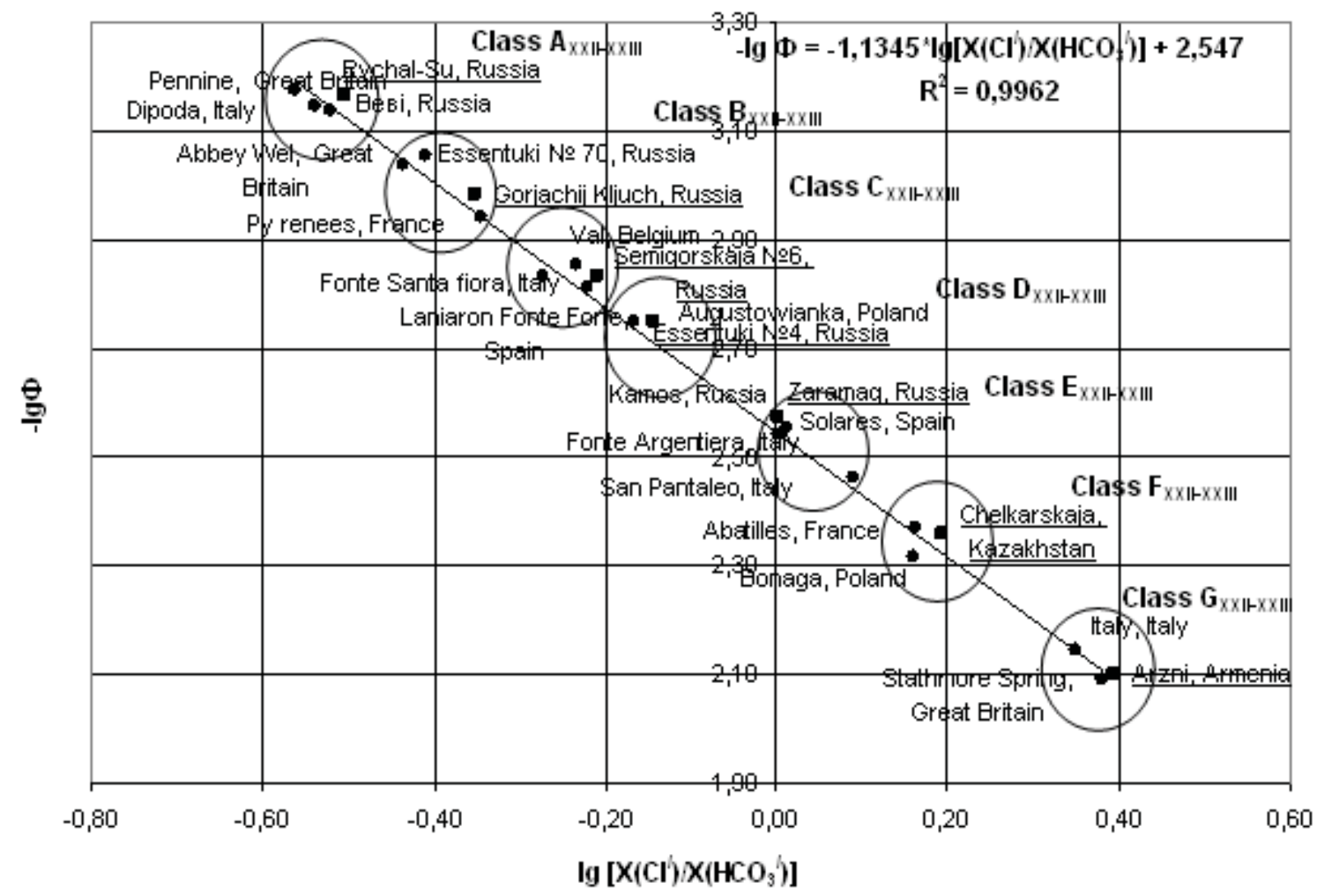

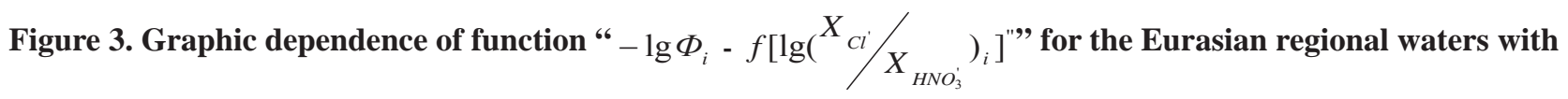
low mineralization. 
At the same time, it is necessary to note that the mineral waters assigned to one class, have various mineralizations, at significant time periods, which in our opinion is due to various conditions of the physical and chemical processes proceeding at their formation and various sources of receipt in water-containing layers $\mathrm{H}_{2} \mathrm{O}_{\text {and }} \mathrm{CO}_{2}$ [2-4].

\section{Conclusions}

As a result of the estimation of structure of Eurasian natural mineral waters and waters with low mineralization of Chloride-Hydrocarbonate type, using the characteristic parameters $\bar{\ni}, B, \Phi$ and $R$, the following conclusions may be drawn.

a) Characteristic parameters $\bar{\ni}, B, \Phi$ and $R$ allow to perform the identification of any mineral water on conformity to reference waters, regardless of the geographical location of the source, with a high degree of reliability;

b) Values of characteristic parameters of the mineral water counted on initial data of the chemical analysis of structure, can serve as a quantitative estimation of quality of mineral water and may be used for the organization of the system of national and interstate waters monitoring;

c) Characteristic parameters of waters structure can be used for the examination of quality and conformity of mineral water to the passport characteristics of declared analogue of standard water, and also for the establishment of source location;

d) The expansion of geography of finding the sources of the mineral waters identified on their conformity to reference mineral waters on characteristic parameters $\vec{Y}, B, \Phi$ and $R$ allows rational utilization of regional natural water resources and promote the social development of territory of location the sources of mineral waters which were disregarded before.

\section{References}

[1]. Kornilov N., Ermolenko O., Kornilova E., (2004), ECE. Ecology. Culture. Education., 13, 28-31.

[2]. Ermolenko O., Kornilov N., Kornilova E., (2008), Indentification Eurasian mineral Chloride-Hydrocarbonate Sodium Waters. Natural\&Technical Sciences., vol.2, 232-234.

[3]. Kornilov N., Ermolenko O., Kornilova E., (2004), Modeling of salty-water systems of the natural waters used the processing industry. Proc. Intern. Sem. on Food industry: integration of science and education, vol.1, 82-83.

[4]. Kornilova E., Ermolenko O., Kornilov N., Kalugin D., (2004), Genesis, classification and modelling Euroasian natural Chloride-Hydrocarbonate waters. Forum on Innovative technologies of XXI century for rational Nature management, Ecology and sustainable development, vol.1, 280-290. 